\title{
RECENT RESULTS FROM THE H1 EXPERIMENT AT HERA
}

\author{
Hans-Ulrich Martyn* \\ I. Physikalisches Institut, RWTH Aachen \\ D-52056 Aachen, Germany
}

\begin{abstract}
Some recent results from the $\mathrm{H} 1$ experiment at the HERA $e p$ collider are presented, based on data taken in 1993 with an integrated luminosity of $\sim 0.5 \mathrm{pb}^{-1}$. The topics include deep inelastic scattering (DIS) of neutral and charged currents, photoproduction, and searches for new particles beyond the Standard Model.

The analysis of the proton structure function $\mathcal{F}_{2}\left(x, Q^{2}\right)$ from neutral current DIS in the novel kinematic region of $3.5 \leq Q^{2} \leq 3000 \mathrm{GeV}^{2}$ and $10^{-4} \leq x \leq 10^{-1}$ confirms the previously observed steep rise towards low $x$ values. Several inclusive and topological measurements of the hadronic final state are presented to extract the gluon density in the proton and to get information on the onset of the BFKL evolution.

A sizeable fraction of DIS events are diffractive with a large rapidity gap between the current and proton remnant. Their properties, contribution to the proton structure function, and interpretation in terms of a Pomeron exchange will be discussed.

Multijet rates in DIS events are investigated and used to demonstrate the running of the strong coupling constant $\alpha_{s}\left(Q^{2}\right)$ and to determine its value at the $Z$ pole.

The charged current reaction $e^{-} p \rightarrow \nu_{e} X$ has been measured and exhibits for the first time the effect of the $W$ propagator.

The scattering of almost-real photons on protons reveals the structure of the photon. Results on direct and resolved $\gamma p$ processes, hard diffractive $\gamma p$ scattering, and charm production will be discussed.

Finally, new limits on phenomena beyond the Standard Model will be given. They include direct searches for heavy leptons, leptoquarks, leptogluons, $R_{p}$-violatinq squarks, and indirect searches via contact interactions.
\end{abstract}

\footnotetext{
*Mailing address: DESY, Notkestr. 85, D-22603 Hamburg, Germany;

e-mail: f34mar@dsyibm.desy.de.
}

(c)H.-U. Martyn 1994 


\section{Introduction}

The e p collider HERA at DESY, in which $27.5 \mathrm{GeV}$ electrons collide with $820 \mathrm{GeV}$ protons, gives access to a so-far-unexplored new kinematic region of lepton nucleon scattering with a rich physics potential. Due to the large center-of-mass energy of $\sqrt{s} \simeq 300 \mathrm{GeV}$, the kinematic phase space rises the observable momentum transfers $Q^{2}$ up to $Q_{\text {max }}^{2} \simeq$ few $10^{4} \mathrm{GeV}^{2}$ by about two orders of magnitude and lowers the detectable Bjorken $x$ values to $x_{\min } \leq 10^{-4}$ at small $Q^{2}$ by about one order of magnitude with respect to fixed target experiments. Furthermore, $\gamma p$ interactions can be studied over a wide center-of-mass energy range of $W_{\gamma p} \simeq 60 \div 270 \mathrm{GeV}$.

HERA was commissioned in 1992 with an initial electron energy of $26.7 \mathrm{GeV}$. The two experiments $\mathrm{H} 1$ and ZEUS published the first interesting physics results based on an integrated luminosity of $\mathcal{L}=0.025 \mathrm{pb}^{-1}$. In 1993, an improved HERA performance with more colliding bunches allowed us to collect about $\mathcal{L}=0.5 p b^{-1}$ per experiment for useful physics analysis. This year, the lepton energy was raised to $27.5 \mathrm{GeV}$. Since the lifetime of the electron beam turned out to be a severe limitation, HERA has operated with positrons since July 18, 1994. A peak

luminosity of $L_{\text {peak }}=4.3 \cdot 10^{30} \mathrm{~cm}^{-2} \mathrm{~s}^{-1}$ is now achieved, about $30 \%$ of its design value. With the increased $e^{+}$lifetime (factor of $\sim 2.5$ ) and a better understanding of the machine as well as the $\mathrm{H} 1$ detector, an integrated luminosity of $\mathcal{L} \simeq 4 p b^{-1}$ for physics analysis can be expected for the current data-taking period.

The topics to be discussed in this talk are a personal selection of the many H1 results from the data-taking period of 1993 presented elsewhere* this summer.

\section{The H1 Detector}

The $\mathrm{H} 1$ detector is a large, multipurpose facility, designed to provide an almosthermetic coverage to detect the final states of e $p$ scattering: the hadronic energy flow, charged particles and precise lepton (electron and muon) identification, and measurement. While the detector is isotropic in azimuth, it extends more towards the forward region (proton and $+z$ direction) in order to accommodate the moving center-of-mass system due to the highly asymmetric proton and electron beam

* Contributions to the International Conference on High Energy Physics, Glasgow, July 20 - 27, 1994. 
energies (see Fig. 1). Details of the H1 detector can be found elsewhere. ${ }^{1}$ Some parameters and actual performance figures are given in Table 1.

Figure 1: Schematic side view of the H1 detector. The luminosity detector, downstream from the electron beam, is not to scale. Protons move to the left $(+z$ direction).

Major emphasis has been given to the calorimetry. The backbone of the detector is a highly transversal and longitudinal segmented liquid argon (LAr) calorimeter. It consists of an electromagnetic and a hadronic section with lead and steel absorbers, respectively. The good hadron energy resolution is achieved by energy weighting techniques during software analysis. The LAr calorimeter provides a very stable response. The absolute energy scales, as determined from test measurements and DIS events, are presently known to $3 \%$ (electromagnetic) and $6 \%$ (hadronic). A lead-scintillator sandwich calorimeter in the backward region (BEMC) serves mainly to identify and measure the scattered electron in low $Q^{2}$ deep inelastic scattering events. A copper-silicon calorimeter (PLUG) covers 


\section{Calorimetry}

\begin{tabular}{|c|c|c|}
\hline Liquid Argon Calorimeter & \multicolumn{2}{|c|}{$4^{\circ}<\theta<153^{\circ}$} \\
\hline granularity (em/hadron) & $10 \div 100 \mathrm{~cm}^{2}$ & $50 \div 2000 \mathrm{~cm}^{2}$ \\
\hline depth (em/hadron) & $20 \div 30 X_{0}$ & $4.7 \div 7 \lambda_{a b s}$ \\
\hline resolution $\sigma(E) / E$ (em/hadron) & $0.12 / \sqrt{E_{e}} \oplus 0.01$ & $\sim 0.50 / \sqrt{E_{h}} \oplus 0.02$ \\
\hline BEMC: Pb-scintillator & \multicolumn{2}{|c|}{$151^{\circ}<\theta<177^{\circ}$} \\
\hline depth - resolution $\sigma\left(E_{e}\right) / E_{e}$ & $22.5 X_{0}\left(1 \lambda_{a b s}\right)$ & $0.10 / \sqrt{E_{e}} \oplus 0.02$ \\
\hline Tail Catcher: streamer tubes & \multicolumn{2}{|c|}{$4^{\circ}<\theta<177^{\circ}$} \\
\hline depth - resolution $\sigma\left(E_{h}\right) / E_{h}$ & $4.5 \lambda_{a b s}$ & $1.0 / \sqrt{E_{h}}$ \\
\hline Plug Calorimeter: $\mathrm{Cu}-\mathrm{Si}$ & \multicolumn{2}{|c|}{$0.7^{\circ}<\theta<3.3^{\circ}$} \\
\hline depth - resolution $\sigma\left(E_{h}\right) / E_{h}$ & $4.25 \lambda_{a b s}\left(45 X_{0}\right)$ & $\sim 1.5 / \sqrt{E_{h}}$ \\
\hline$e, \gamma$ Tagger: $\mathrm{Tl}(\mathrm{Cl} / \mathrm{Br})$ & \multicolumn{2}{|c|}{$\theta>179.7^{\circ}$} \\
\hline depth - resolution $\sigma\left(E_{e}\right) / E_{e}$ & $21 X_{0}$ & $\sim 0.10 / \sqrt{E_{e}} \oplus 0.01$ \\
\hline \multicolumn{3}{|c|}{ Tracking } \\
\hline Central Tracking & \multicolumn{2}{|c|}{$25^{\circ}<\theta<155^{\circ}$} \\
\hline spatial resolution & $\sigma_{r \phi}=170 \mu m$ & $\sigma_{z}=2 \mathrm{~mm}$ \\
\hline momentum $-d E / d x$ resolution & $\sigma_{1 / p}<0.01 \mathrm{GeV}^{-1}$ & $\sigma_{d E / d x}=10 \%$ \\
\hline Forward/Backward Tracking & $7^{\circ}<\theta<25^{\circ}$ & $155^{\circ}<\theta<175^{\circ}$ \\
\hline spatial resolutions & $\sigma_{r \phi}=170 \mu m$ & $\sigma_{\theta}<5 \mathrm{mr}$ \\
\hline & $\sigma_{x, y}=210 \mu m$ & $\sigma_{x, y}=1 \mathrm{~mm}$ \\
\hline \multicolumn{3}{|c|}{ Muon Detection } \\
\hline Instrumented Iron & \multicolumn{2}{|c|}{$4^{\circ}<\theta<171^{\circ}$} \\
\hline resolutions (barrel) & $\sigma_{r \phi}=3.5 \mathrm{~mm}$ & $\sigma_{z}=12.5 \mathrm{~mm}$ \\
\hline & $\sigma_{p} / p \approx 0.35$ & $\sigma_{\theta}\left(\sigma_{\phi}\right)=15(10) m r$ \\
\hline Forward Muon Toroid & \multicolumn{2}{|c|}{$3^{\circ}<\theta<17^{\circ}$} \\
\hline momentum resolution - range & $\sigma_{p} / p \sim 0.25$ & $5 \div 200 \mathrm{GeV}$ \\
\hline
\end{tabular}

Table 1: Parameters and performance of the $\mathrm{H} 1$ detector. 
the very forward regions. All calorimeters are placed inside a superconducting coil in order to minimize the inactive material in front of them. The flux return yoke is instrumented with streamer tubes (analogue readout) to measure the leakage of hadronic showers (Tail Catcher).

Charged particle tracks are measured in the central and forward tracking systems placed inside a uniform axial magnetic field of $1.15 \mathrm{~T}$. Each consists of a hybrid of drift and proportional chambers, providing good space information in orthogonal directions as well as some particle identification $(d E / d x$ and transition radiation).

Muon detection and measurement is performed with streamer tubes in the instrumented iron and a dedicated toroidal spectrometer in the forward direction.

A scintillator hodoscope behind the BEMC is used to veto proton-induced background based on its early arrival compared to genuine e p interactions.

The luminosity is determined from the Compton process e $p \rightarrow e p \gamma$ at very small scattering angles. The electron tagger (ET) and photon detector (PD) are situated far downstream the electron direction (at $-33 \mathrm{~m}$ and $-103 \mathrm{~m}$ ) and serve as well to detect photoproduction events. At present, the systematic uncertainty of the luminosity measurement amounts to $5 \%$.

\section{Kinematics}

The lowest order diagram in the quark-parton model (QPM) of the neutral current $(\mathrm{NC})$ or charged current (CC) process $e p \rightarrow e^{\prime}(\nu) X$ is sketched in Fig. 3, where the four vectors involved in the kinematics are also defined.

At HERA, electrons of $26.7 \mathrm{GeV}$ collide against protons of $820 \mathrm{GeV}$, giving a total center-of-mass $(\mathrm{cms})$ energy of $\sqrt{s}=296 \mathrm{GeV}$. The kinematics are determined by two independent variables, conventionally chosen from $Q^{2}$, the squared momentum transfer of the exchanged boson, Bjorken $x$, the momentum fraction of the proton carried by the struck parton, and $y$, the energy fraction transferred from the incoming lepton to the hadronic system in the proton rest frame. These quantities are related via $Q^{2}=x y s$. The Lorentz invariant kinematic variables are defined as follows:

$$
\begin{aligned}
s & =4 E_{e} E_{p} \\
Q^{2} & =-\left(p_{e}-p_{e}^{\prime}\right)^{2}=-q^{2}
\end{aligned}
$$


Figure 2: Lowest order diagram (QPM) for $e p \rightarrow e^{\prime}(\nu) X$ and definition of four vectors.

$$
\begin{aligned}
& y=\frac{P \cdot q}{P \cdot p_{e}} \\
& x=\frac{Q^{2}}{2 P \cdot q} .
\end{aligned}
$$

Since the H1 detector has an almost hermetic $4 \pi$ coverage, the kinematics can be determined using information from either the scattered lepton or the hadronic final state, or a mixture of both (see, e.g., Ref. 2). This redundancy allows us to make experimental cross-checks and to choose the optimum resolution. The following kinematic reconstruction methods are used in DIS NC analyses.

1. The electron method:

$$
\begin{aligned}
y_{e} & =1-\frac{E_{e}^{\prime}}{E_{e}} \sin ^{2} \frac{\theta_{e}}{2} \\
Q_{e}^{2} & =4 E_{e} E_{e}^{\prime} \cos ^{2} \frac{\theta_{e}}{2}
\end{aligned}
$$

$Q_{e}^{2}$ is always well-measured. This method provides good resolutions in a large kinematic region except at low $y$ (high $x$ ). The electron method is preferred at high $y>0.15$.

2. The $\Sigma$ method:

$$
\begin{aligned}
y_{\Sigma} & =\frac{\sum_{h}\left(E_{h}-p_{z h}\right)}{\sum_{e, h}\left(E-p_{z}\right)} \\
Q_{\Sigma}^{2} & =\frac{p_{T e}^{2}}{1-y_{\Sigma}}
\end{aligned}
$$


The use of the quantity $\sum_{e, h}\left(E-p_{z}\right) \simeq 2 E_{e}(h=$ hadrons $)$ for DIS NC partially removes the large initial state radiative corrections present in the electron method. The resolutions are good at low $y$ (high $x$ ) and still reasonable at low $x$. The $\Sigma$ method is preferred at low $y<0.15$.

3. The double-angle method:

$$
\begin{aligned}
Q_{d a}^{2} & =\frac{4 E_{e}^{2} \sin \gamma\left(1+\cos \theta_{e}\right)}{\sin \gamma+\sin \theta_{e}-\sin \left(\gamma+\theta_{e}\right)} \\
x_{d a} & =\frac{E_{e}}{E_{p}} \cdot \frac{\sin \gamma+\sin \theta_{e}+\sin \left(\gamma+\theta_{e}\right)}{\sin \gamma+\sin \theta_{e}-\sin \left(\gamma+\theta_{e}\right)} .
\end{aligned}
$$

This method is independent of the absolute energy scales, since only the directions of the electron, $\theta_{e}$, and the "struck quark" in the QPM, $\gamma$, are used, where

$$
\cos \gamma=\frac{\left(\sum_{h} p_{x h}\right)^{2}+\left(\sum_{h} p_{y h}\right)^{2}-\left(\sum_{h}\left(E_{h}-p_{z h}\right)\right)^{2}}{\left(\sum_{h} p_{x h}\right)^{2}+\left(\sum_{h} p_{y h}\right)^{2}+\left(\sum_{h}\left(E_{h}-p_{z h}\right)\right)^{2}} .
$$

The resolutions are comparable to the $\Sigma$ method. The double-angle method primarily serves as a cross-check.

Experimentally, DIS NC events are rather arbitrarily defined as having a scattered electron detected in the central calorimeters, i.e., $\theta_{e} \leq 175^{\circ}$ or $Q^{2} \geq 5 \mathrm{GeV}^{2}$. The bulk of the data, however, are photoproduction events with a huge cross section, where the electron is scattered under large angles into the backward direction. For tagged electrons, $\theta_{e}>179.7^{\circ}$ and $Q^{2} \simeq 3 \cdot 10^{-8} \div 10^{-2} \mathrm{GeV}^{2}$. The relevant kinematic variables are measured from

$$
\begin{aligned}
y & =\frac{E_{\gamma}}{E_{e}}=1-\frac{E_{e}^{\prime}}{E_{e}}, \\
W_{\gamma p} & =\sqrt{y s} .
\end{aligned}
$$

Besides the interest in the rather inclusive quantities $Q^{2}, x$, and $y$, many analyses are concerned with topological event properties, such as jet rates and jet properties. Applying any jet algorithm, events are conveniently classified as $N+1$ jet events. The +1 jet accounts for the proton remnant fragments, of which only a small part is detectable in the calorimeters around the beam pipe. Topologies with $1+1$ jets arise from the QPM diagram of Fig. 3 , while $2+1$ jet configurations may originate from the $\mathcal{O}\left(\alpha_{s}\right)$ processes of boson gluon fusion [BGF, Fig. 3(a)] and QCD Compton scattering [(Fig. 3(b)]. The number of observed $N$ jets depends 
Figure 3: DIS NC processes of $\mathcal{O}\left(\alpha_{s}\right)$ leading to $2+1$ jet configurations for (a) boson gluon fusion (BGF), and (b) QCD Compton.

strongly on the jet definition, and respectively, the jet algorithm and resolution parameters used. In $2+1$ jet events, the momentum fraction $x_{\text {parton }}$ of the struck parton in the proton can be reconstructed from the invariant mass $m_{j j}$ of the two observed jets

$$
x_{\text {parton }}=x\left(1+\frac{m_{j j}^{2}}{Q^{2}}\right) .
$$

\section{The Structure of the Proton}

\subsection{The Proton Structure Function $\mathcal{F}_{2}\left(x, Q^{2}\right)$}

Neglecting the $Z$ boson exchange, which does not contribute significantly at the present integrated luminosity, the differential cross section for the DIS NC process $e p \rightarrow e^{\prime} X$ can be expressed in terms of the proton structure function $\mathcal{F}_{2}\left(x, Q^{2}\right)$

$$
\frac{\mathrm{d}^{2} \sigma}{\mathrm{d} x \mathrm{~d} Q^{2}}=\frac{2 \pi \alpha^{2}}{x Q^{4}}\left\{2(1-y)+\frac{y^{2}}{1+R}\right\} \mathcal{F}_{2}\left(x, Q^{2}\right) .
$$

The ratio $R=\sigma_{L} / \sigma_{T}$ is not yet measured at HERA, and therefore is taken from the QCD prescription.

The event selection is based on an integrated luminosity of $\mathcal{L}=271 n b^{-1}$ and

covers the kinematic region $3.5 \mathrm{GeV}^{2} \leq Q^{2} \leq 3000 \mathrm{GeV}^{2}$ and $10^{-4} \leq x \leq 0.2$. Several independent analyses have been performed using the electron method, the $\Sigma$ method, and the double-angle method to reconstruct the kinematics. All analyses agree with each other. The final results are obtained from a combination of the electron and $\Sigma$ methods, optimizing the kinematic resolutions, and minimizing the bin-to-bin migrations. The data are corrected for detector and 
radiative effects using a Monte Carlo ${ }^{3}$ (LEPTO matrix elements + parton showers + QED radiation) with the MRS $\mathrm{H}^{4}$ input structure function. The overall systematic uncertainty on the extracted structure function amounts to $\sim 10 \%$ from the uncertainty in the calorimeter energy scales and 5\% from the luminosity measurement (not included in the figures).

Figure 4: The proton structure function $\mathcal{F}_{2}\left(x, Q^{2}\right)$ as a function of $x$ for several $Q^{2}$ intervals. The $\mathrm{H} 1$ data $(\bullet)$ are compared with $\operatorname{NMC}(\circ)$ and $\operatorname{BCDMS}(\triangle)$ measurements. The curves are results of a QCD fit.

The final results on $\mathcal{F}_{2}\left(x, Q^{2}\right)$ are presented in Fig. 4 as a function of $x$ for various $Q^{2}$ bins. The $Q^{2}$ dependence of the proton-structure function at fixed $x$ intervals is shown in the traditional plot of Fig. 5. The H1 data are in good agreement with the ZEUS results. ${ }^{5}$ They are also in agreement with the low energy experiments $\mathrm{NMC}^{6}$ and $\mathrm{BCDMS}^{7}$ in the region of overlap. Note the enormous extension of the kinematic phase space with respect to fixed target experimentsabout two orders of magnitude towards higher $Q^{2}$ as well as towards lower $x$ values at small $Q^{2}$. 
Figure 5: The proton structure function $\mathcal{F}_{2}\left(x, Q^{2}\right)$ as a function of $Q^{2}$ for different $x$ intervals, together with data from NMC and BCDMS. The curves are results of a QCD fit. The scale of the $\mathcal{F}_{2}$ axis has been multiplied by alternating factors of three and ten when going from the highest $x$ bin to lower $x$ bins.

A striking feature is the dramatic rise of $\mathcal{F}_{2}\left(x, Q^{2}\right)$ at low $x$ seen in Fig. 4, which confirms earlier observations at HERA. ${ }^{8,9}$ This rise is attributed to an increased gluon density (and consequently, a growing sea quark density) at low $x$ values. Several models have been proposed to describe $\mathcal{F}_{2}\left(x, Q^{2}\right)$ as follows:

- One approach is to assume a flat gluon density $x g\left(x, Q_{0}^{2}\right) \stackrel{x \rightarrow 0}{\sim}$ const., like MRS D ${ }^{0}$ and CTEQ1, ${ }^{10}$ or a Regge behavior. ${ }^{11}$ These structure functions generally fall below the data and do not exhibit the observed strong rise.

- Another approach is to assume a rising gluon density $x g(x) \sim x^{-\lambda}$, like MRS H, MRS D-, and CTEQ2, but otherwise take the usual DGLAP ${ }^{12}$ parton evolutions. Such a singular behavior is a pure phenomenological parametrization of the gluon put in by hand. It is motivated by the BFKL ${ }^{13}$ 
evolution predicting $\lambda=0.5$, in which the summation of large $\log (1 / x)$ contributions is taken into account (see Sec. 4.3). These structure functions are able to reproduce the data by a proper choice of $\lambda \sim 0.3$.

- A third approach is to start with input parton distributions at a very low $Q_{0}^{2}$ scale and to evolve them in a double-leading logarithm approximation into the HERA regime. An example is the $\mathrm{GRV}^{14}$ structure function, which describes the data reasonably well.

A leading order QCD fit ${ }^{15}$ has been performed using the DGLAP parton evo-

lution equations with $\Lambda_{Q C D}=240 \mathrm{MeV}$ and four flavors. The parton densities have the form $x f(x) \sim x^{\beta}(1-x)^{\gamma}(1+\delta x+\epsilon \sqrt{x})$. The high $x$ parameters are taken from low-energy data as given by the MRS H parton densities, while the low $x$ parameters $\beta$ and the normalizations are treated as free parameters. The results of the fit are shown as curves in Figs. 4 and 5, and demonstrate that the data are well-described by perturbative QCD.

These new proton structure function measurements in a novel kinematic region will lead to the determination of more reliable parton densities and, in addition, may be used for the calculation of cross sections at very high energy hadron colliders.

\subsection{The Gluon Density in the Proton}

In order to get insight into the gluon density in the proton, several methods have been investigated. One can either use the inclusive measurement of the proton structure function or study the rate of boson gluon fusion processes.

- The leading order QCD fit to $\mathcal{F}_{2}\left(x, Q^{2}\right)$ described in Sec. 4.1 gives the following result for the gluon density and its normalization:

$$
\begin{aligned}
x g(x) & \sim x^{-(0.38 \pm 0.08)} \\
\int x g(x) d x & =0.57 \text { at } Q_{0}^{2}=5 \mathrm{GeV}^{2} .
\end{aligned}
$$

The value of the $x$-exponent of $\beta_{g}=-0.38 \pm 0.08$ is close to the one favored by the BFKL evolution. However, it cannot be concluded that this mechanism is the only explanation for the steep rise of the gluon distribution.

- Using the fact that the $\mathcal{F}_{2}\left(x, Q^{2}\right)$ scaling violations at low $x \leq 10^{-2}$ are dominated by the conversion of gluons into $q \bar{q}$ pairs, one can relate the 
gluon density directly to the $Q^{2}$ dependence of the proton structure function. Prytz $^{16}$ derives the following approximation in leading order, from which the gluon density can be extracted

$$
\frac{\partial \mathcal{F}_{2}\left(x, Q^{2}\right)}{\partial \ln Q^{2}} \simeq \frac{20 \alpha_{s}\left(Q^{2}\right)}{27 \pi} x g\left(2 x, Q^{2}\right)
$$

- A different approach is to analyze boson gluon fusion events with a $2+1$ jet topology and jet-jet masses $m_{j j}>10 \mathrm{GeV}$. The $x_{\text {parton }}$ of the struck parton in the proton is reconstructed from Eq. (14). Unfolding the $x_{\text {parton }}$ distribution and correcting for QCD Compton background ( 20\%) and migrations $(\sim 10 \%)$, one gets the gluon distribution in the proton. Due to the event selection, this method gives only access to $x_{g} \geq 5 \cdot 10^{-3}$.

The results of the three analyses are consistent with each other and are displayed in Fig. 6. Despite the still large errors, the data clearly prefer a rising gluon density with decreasing $x$, as already anticipated by the proton structure function behavior.

Figure 6: The gluon distribution $x g(x)$ in the proton as function of $x$. 


\subsection{Evidence for BFKL Evolution?}

Many QCD aspects of deep inelastic lepton nucleon scattering are adequately described by the DGLAP ${ }^{12}$ evolution equations. However, it is questionable whether this prescription still holds in the low $x$ region accessible at HERA or has to be supplemented by another parton evolution model. One approach to describe the low $x$ phenomena is the BFKL ${ }^{13}$ evolution.

Figure 7: Ladder diagram of deep inelastic e $p \rightarrow e^{\prime} X$ scattering.

The basic diagram to discuss the features of the DGLAP and BFKL schemes is illustrated in Fig. 7. Before the struck parton hits the virtual photon $\left(x=x_{B j}\right)$ to produce a current jet (labeled as $n$ ), it may emit a number of gluons.

In the DGLAP scheme, the evolution proceeds in $\alpha_{s} \log Q^{2}$. The fractional momenta of the gluons decrease along the ladder, $x \leq x_{n} \leq \ldots \leq x_{1}$, while the transverse momenta increase and are strongly ordered, $Q^{2} \gg k_{\perp n}^{2} \gg \ldots \gg k_{\perp 1}^{2}$. As a consequence, the transverse energy flow $E_{T}$ will be depleted between the current and proton, and enhanced towards the current direction.

At small $x \leq 10^{-2}$, large $\log (1 / x)$ terms are encountered, which need to be resummed. This is done in the BFKL scheme, where the evolution proceeds in $\alpha_{s} \log (1 / x)$. The longitudinal momenta of the gluons along the ladder still decrease, $x \ll x_{n} \ll \ldots \ll x_{1}$; however, no ordering of the transverse momenta $k_{\perp i}$ is imposed as they undergo a "random walk." The diffusion of transverse momenta leads to a reduction of the $E_{T}$ flow around the current, being shifted towards the proton remnant. Another outcome of the BFKL evolution is the prediction of a steeply rising gluon density $x g(x) \sim x^{-\lambda}$ with $\lambda=0.5$. 
Figure 8: The mean transverse energy flow $E_{T}$ as a function of pseudorapidity $\eta$ at $x<10^{-3}$ and $\left\langle Q^{2}\right\rangle \simeq 15 \mathrm{GeV}^{2}$. The proton direction is to the right. (a) The H1 data compared to various Monte Carlo models including hadronization. (b) The same data compared to an analytic calculation in the BFKL scheme. The histogram indicates the effects of hadronization in the MEPS model.

Thus, the most sensitive kinematic phase space to study the onset of BFKL dynamics is the region between the current and proton remnant, $x \ll x_{j} \ll 1$.

\subsubsection{Transverse Energy Flow "Away" from the Current Jet ${ }^{17}$}

Figure 8(a) shows the mean transverse energy flow $E_{T}$ as a function of the pseudorapidity $\eta=-\ln \tan (\theta / 2)$ in the laboratory system for low $\langle x\rangle \simeq 5.7 \cdot 10^{-4}$ and moderate $\left\langle Q^{2}\right\rangle \simeq 15 \mathrm{GeV}^{2}$. The energy flow exhibits an enhancement around the current direction in the central region and levels off at about $2 \mathrm{GeV}$ per unit of rapidity towards the proton remnant at large positive $\eta$ values. The MEPS model (matrix elements plus parton showers), which uses the DGLAP evolution, produces considerably less energy in the forward region than observed. The color dipole model ${ }^{3}(\mathrm{CDM})$ with quite different dynamics, which supposedly mimics the BFKL behavior to some extent, is closer to the data.

Recently, analytic calculations at the parton level of the $E_{T}$ flow using the BFKL scheme have been performed, ${ }^{18}$ shown in Fig. 8(b). As an illustration, the hadronization contribution to the $E_{T}$ flow is shown for the MEPS model. However, it is not obvious how the model-dependent, nonperturbative hadronization affects the calculations, whether it can just be added. Despite this, the BFKL expectation 
of the transverse energy flow "away" from the current jet is much closer to the data than the expectations from DGLAP-based models.

\subsubsection{Events with an Energetic Forward Jet}

Another "footprint" for BFKL dynamics may be obtained from the rate of events with an energetic forward jet. ${ }^{19,20}$ The sensitivity to the BFKL scheme is enhanced for jets with $k_{\perp j e t}^{2} \simeq Q^{2}$ and $x \ll x_{j e t} \ll 1$, where little phase space is left in the DGLAP type evolution (see Fig. 7). Energetic forward jets are defined by a cone algorithm of radius $R=\sqrt{\Delta \eta^{2}+\Delta \phi^{2}}=1$ and $p_{T, \text { jet }}>5 \mathrm{GeV}$ within $5^{\circ}<\theta_{\text {jet }}<20^{\circ}$. DIS events are selected by requiring $x<2 \cdot 10^{-3}, x_{\text {jet }}>0.05$, and $0.5<p_{T, j e t}^{2} / Q^{2}<6$ at an average $Q^{2} \simeq 20 \mathrm{GeV}^{2}$.

\begin{tabular}{ccccc}
\hline$x$ range & Data & MEPS & $\begin{array}{c}\text { BFKL } \\
\text { analytic }\end{array}$ & $\begin{array}{c}\text { DGLAP } \\
\text { analytic }\end{array}$ \\
\hline $2 \cdot 10^{-4} \div 2 \cdot 10^{-3}$ & $128 \pm 12 \pm 26$ & 69 & 111 & 45 \\
$2 \cdot 10^{-4} \div 1 \cdot 10^{-3}$ & $85 \pm 9 \pm 17$ & 37 & 75 & 25 \\
$1 \cdot 10^{-3} \div 2 \cdot 10^{-3}$ & $43 \pm 7 \pm 17$ & 32 & 36 & 20
\end{tabular}

Table 2: The uncorrected rates of forward jets observed and expected for an integrated luminosity of $\mathcal{L}=330 \mathrm{nb}^{-1}$. MEPS denotes a full detector Monte Carlo including hadronization. BFKL and DGLAP refer to analytic calculations at parton level.

The number of observed events with an energetic forward jet is given in Table 2 together with some model predictions. ${ }^{20}$ The data show a clear increase in the rates by a factor of $\sim 2$ as $x$ decreases. It is rather the $x$ dependence which should be compared to model predictions, since the absolute values are less reliable. The DGLAP-based models - the full MEPS simulation including hadronization and its analytical variant at the parton level - badly fail to describe the observed rise at low $x$. On the other hand, the analytic BFKL calculation shows a similar $x$ dependence as observed in the data.

Whether these two observations - the enhanced transverse energy flow between the current and proton remnant, and the rate of events with an energetic forward jet at low $x$ values - can be regarded as evidence for the onset of BFKL dynamics 
remains still open. They are certainly interesting first signs for a breakdown of conventional DGLAP evolution. More detailed studies are required, and in particular, improved Monte Carlo event generators including the BFKL evolution scheme are needed.

\subsection{Diffractive Scattering}

It has been argued that HERA provides a rather unique possibility to study diffractive dissociation at short distances (see, e.g., HERA workshop ${ }^{2}$ ). Quoting Bjorken: "A diffractive process occurs if and only if there is a large rapidity gap in the produced particle phase space which is not exponentially suppressed." A method to discriminate diffractive scattering against conventional DIS is to classify events according to the maximum pseudorapidity $\eta_{\max }$, i.e., closest to the proton direction, at which some energy $E_{\min }$ is deposited in the detector.

Figure 9: Distribution of events as a function of $\eta_{\max }$ with $E_{\min }>0.4 \mathrm{GeV}$ for DIS events. (a) Comparison of data with LEPTO and VMD expectations. (b) Comparison of data with LEPTO and RAPGAP expectations.

The $\eta_{\max }$ spectrum of the data ${ }^{21}$ with $E_{\min }>0.4 \mathrm{GeV}$, based on an integrated luminosity of $273 n b^{-1}$, is shown in Fig. 9. The bulk of the data deposit energy in the forward LAr calorimeter, close to its acceptance limit $\eta_{L A r} \sim 3.65$. There is, 
however, a class of events with a large rapidity gap ${ }^{22,23}$ extending into the central and backward region of the detector. Such events are not accounted for in the usual DIS event generators like, e.g., LEPTO.

Figure 10: Diagrams for vector meson $(\rho, \omega, \phi)$ and electron Pomeron scattering. (a) VMD scattering (graphs with vector meson and/or proton dissociation omitted), (b) inelastic $q \mathbb{P}$ scattering, and (c) inelastic $g \mathbb{P}$ scattering.

Assuming that the proton interacts diffractively, the observation of large rapidity gap events can be viewed as the exchange of a colorless Regge pole in the $t$ channel, the Pomeron. Two possible models have been considered in the following analysis. The first mechanism assumes vector meson scattering $\rho, \omega, \phi$, which can be either elastic [Fig. 10(a)] or accompanied by soft dissociation of the vector meson and/or proton (VMD model). The second mechanism considers the possibility that any partonic structure of the Pomeron, a quark [Fig. 10(b)] or a gluon [(Fig. $10 \mathrm{c})]$, is resolved by the highly virtual photon (RAPGAP model ${ }^{24}$ ). Both models account for the rapidity gap phenomena and, when properly combined with the LEPTO generator, are able to describe the $\eta_{\max }$ distribution of Fig. 9.

Experimentally, large rapidity gap events are defined by satisfying $\eta_{\max }<1.8$ (or $\theta>20^{\circ}$ ) for $E_{\min }>0.4 \mathrm{GeV}$. Some of their properties can be summarized as follows: $:^{21}$

- A fraction of $\sim 5 \%$ of observed DIS events have a large rapidity gap.

This rate is almost independent of $Q^{2}$ at fixed $x$.

The same $Q^{2}$ independence is found for invariant masses $M_{X}>7.5 \mathrm{GeV}$. These observations are consistent with leading twist effects in QCD. 
- The invariant mass spectrum peaks at low $M_{X}$ compared to DIS.

- Both the VMD and the RAPGAP model describe the properties of the large rapidity gap events equally well. However,

- about $10 \%$ of the events are exclusive vector meson production,

- a significant fraction of events has a jet and even two-jet structure.

Thus, a definite interpretation of the diffractive processes is still open.

The occurence of large rapidity gap events cannot explain the rise of the proton structure function $\mathcal{F}_{2}\left(x, Q^{2}\right)$ at low $x$. A structure function analysis has been done for the large rapidity gap events, restricting the $x$ values of the Pomeron to $x_{I P} \leq 10^{-2}$ as imposed by the selection criteria. The diffractive contribution to $\mathcal{F}_{2}\left(x, Q^{2}\right)$ is displayed in Fig. 11 for the low $Q^{2}$ data-it is an order of magnitude smaller than the total $\mathcal{F}_{2}$.

Figure 11: Diffractive contribution to the proton structure function $\mathcal{F}_{2}\left(x, Q^{2}\right)$ for $x_{I P} \leq 10^{-2}$ in the low $Q^{2}$ data. 
An attempt has been made to investigate whether the diffractive scattering can be factorized into a flux factor describing the momentum distribution $x_{\mathbb{P}}$ of the Pomeron in the proton and a Pomeron-structure function. The differential cross section in terms of a diffractive structure function $F_{2}^{D}\left(x, Q^{2} ; x_{\mathbb{I P}}, t\right)$ and the factorization ansatz can be written as

$$
\begin{aligned}
\frac{\mathrm{d}^{4} \sigma\left(e p \rightarrow e p^{\prime} X\right)}{\mathrm{d} x \mathrm{~d} Q^{2} \mathrm{~d} x_{\mathbb{P}} \mathrm{d} t} & =\frac{4 \pi \alpha^{2}}{x Q^{4}}\left(1-y+\frac{y^{2}}{2}\right) F_{2}^{D}\left(x, Q^{2} ; x_{\mathbb{P}}, t\right), \\
F_{2}^{D}\left(x, Q^{2} ; x_{\mathbb{P}}, t\right) & =f\left(x_{\mathbb{P}}, t\right) F_{2}^{\mathbb{P}}\left(z, Q^{2}\right), \\
z & =\frac{x}{x_{\mathbb{P}}}=\frac{Q^{2}}{Q^{2}+M_{X}^{2}} .
\end{aligned}
$$

Here, $t=\left(P-P^{\prime}\right)^{2}$ is the squared momentum transfer between the incoming and outgoing proton (which, for the time being, cannot yet be measured); $f\left(x_{\mathbb{P}}, t\right)$ describes the Pomeron flux and $F_{2}^{I P}\left(z, Q^{2}\right)$ the Pomeron structure function.

If factorization holds, then $\int F_{2}^{D}\left(x, Q^{2} ; x_{\mathbb{P}}, t\right) \mathrm{d} t=F_{2}^{D}\left(z, Q^{2} ; x_{\mathbb{P}}\right)$ should have the same $x_{\mathbb{P}}$ dependence for any constant $z$ and $Q^{2}$. This conjecture is indeed confirmed by the data. As an example, $F_{2}^{D}\left(z, Q^{2} ; x_{\mathbb{P}}\right)$ as a function of $x_{I P}$ and different $z$ values at $Q^{2}=15 \mathrm{GeV}^{2}$ are shown in Fig. 12. A universal $x_{\mathbb{P}}$ dependence is observed. A global fit to all data $\left(\chi^{2}=1.05\right.$ per degree of freedom) yields

$$
F_{2}^{D}\left(z, Q^{2} ; x_{\mathbb{P}}\right)=x_{\mathbb{P}}^{-(1.3 \pm 0.1)} F_{2}^{\mathbb{P}}\left(z, Q^{2}\right)
$$

The measurements support clear evidence for diffractive production in deep inelastic lepton nucleon scattering, but its interpretation is still debatable. They may provide access to the elusive Pomeron.

\section{$5 \quad$ Jet Rates and $\alpha_{s}\left(Q^{2}\right)$}

The hadronic final state in deep inelastic scattering is an abundant source of jets as illustrated in Fig. 3. The production rate of multijet events is a function of the strong coupling constant $\alpha_{s}\left(Q^{2}\right)$ and of the gluon density in the proton. With the available statistics, it is not possible to determine both quantities simultaneously. In the present analysis, ${ }^{25}$ the $2+1$ jet rate has been measured in order to extract $\alpha_{s}\left(Q^{2}\right)$. The quark and gluon densities are taken from low energy experiments as parametrized in MRS $\mathrm{D}^{-}$or MRS H. 
Figure 12: The diffractive structure function $F_{2}^{D}\left(z, Q^{2} ; x_{\mathbb{P}}\right)$ as a function of $x_{\mathbb{P}}$ for different $z$ values at $Q^{2}=15 \mathrm{GeV}^{2}$. The curves are a universal fit to the data. 
DIS events are classified as having $N+1$ jets using the JADE algorithm and requiring the direction of each jet (except the +1 proton remnant) to be within $10^{\circ}<\theta_{j e t}<145^{\circ}$. A jet resolution parameter is defined as $y_{c}=m_{i j}^{2} / W^{2}$ with $m_{i j}$ being the invariant mass of any two clusters and $W$ the invariant mass of the whole hadronic system. The observed $N+1$ jet rates $R_{N+1}$ as a function of $y_{c}$ is shown in Fig. 13 for two $Q^{2}$ data samples. The MEPS model with the structure function MRS $\mathrm{D}^{-}$gives a very good description of the $R_{N+1}$ distributions in the LAr sample for all $y_{c}$ values. The BEMC sample is fairly described for $y_{c}>0.015$.

Figure 13: Multijet rates $R_{N+1}$ as a function of the jet resolution parameter $y_{c}$ for $Q^{2} \leq 100 \mathrm{GeV}^{2}$ (BEMC sample) and $Q^{2}>100 \mathrm{GeV}^{2}$ (LAr sample). The observed jet rates $(\bullet)$ are compared to MEPS expectations at detector level.

The jet rates strongly depend on the resolution parameter; for further analysis, a value of $y_{c}=0.02$ is chosen. The quantity of interest is the $2+1$ jet rate

$$
\begin{aligned}
R_{2+1}\left(Q^{2}\right) & =\frac{\sigma_{2+1}\left(Q^{2}\right)}{\sigma_{1+1}\left(Q^{2}\right)+\sigma_{2+1}\left(Q^{2}\right)}, \\
& =f\left[\alpha_{s}\left(Q^{2}\right), \text { parton densities }\right] .
\end{aligned}
$$


Figure 14: (a) The $2+1$ jet rate $R_{2+1}$ as a function of $Q^{2}$ compared to PROJET expectations for various $\Lambda$ values. (b) The measured values of $\alpha_{s}$ as a function of $Q^{2}$. The dashed curve shows the fit result to the regularization group equation. For comparison, a fit to the assumption $\alpha_{s}=$ const. is included (dash-dotted line).

The MEPS model is taken to unfold the observed $2+1$ jet rates to the parton level, where they can be compared to the analytic $\mathcal{O}\left(\alpha_{s}^{2}\right)$ calculations of PROJET ${ }^{26}$ using the MRS $\mathrm{D}^{-}$parton densities.

The $2+1$ jet rate and a determination of $\alpha_{\mathcal{S}}\left(Q^{2}\right)$ depend critically on the inclusion of the low $Q^{2}$ data, for which, however, the uncertainty of the parton densities is largest. Note that for $2+1$ jet events, the $x$ values of the struck parton are limited to $x_{\text {parton }}>0.02$ due to the $y_{c}$ choice [see Eq. (14)], while for $1+1$ jet events, $x_{\text {parton }}>1.5 \cdot 10^{-3}$. In order to remove this imbalance in Eq. (19) and to reduce the uncertainties on the parton distributions, only events with $x_{\text {parton }}>0.01$ are further used.

The corrected jet rate $R_{2+1}$ as a function of $Q^{2}$ is shown in Fig. 14(a). The agreement with the PROJET calculations for different values of the QCD parameter $\Lambda$ defined in the $\overline{M S}$ scheme with four flavors is good. The evaluation of the strong coupling constant $\alpha_{s}$ as a function of $Q^{2}$ is presented in Fig. 14(b). There is clear evidence for a running $\alpha_{s}\left(Q^{2}\right)$ from the jet rates, seen for the first time in a single experiment. A constant $\alpha_{s}$ can be ruled out. 
Applying the regularization group equation to extrapolate to $M_{Z}^{2}$, a fit to the data yields a preliminary value for the strong coupling constant

$$
\alpha_{s}\left(M_{Z}^{2}\right)=0.121 \pm 0.009 \text { (stat) } \pm 0.012 \text { (sys) }
$$

where the systematic error includes uncertainties due to detector effects, QCD models, parton densities, the choice of $y_{c}$, and the renormalization and factorization scales. This value compares nicely with $\alpha_{s}\left(M_{Z}^{2}\right)=0.119 \pm 0.003(\exp ) \pm$ 0.010 (theor) as obtained from jet rates by LEP experiments ${ }^{27}$ and demonstrates the consistency of the underlying QCD picture.

\section{The Charged-Current Cross Section}

In the past decades, the weak charged-current has been extensively studied in $\nu N$ scattering experiments. ${ }^{28}$ The total cross section is found to rise linearly with the neutrino energy. However, the energies are far too low to be sensitive to any $W$ propagator effect, as seen in Fig. 16. The first observation of the charged-current reaction $e p \rightarrow \nu X$ at HERA ${ }^{29}$ opens a new, rich field of electroweak physics. This process is just the inverse of $\nu N$ scattering, but at energies equivalent to $\sim 50 \mathrm{TeV}$ in fixed target experiments.

The charged-current analysis ${ }^{29}$ is extremely simple and involves almost no Monte Carlo! The signature is a large missing transverse momentum carried by the undetected neutrino, $\vec{p}_{\perp}^{\text {miss }}=\vec{p}_{\perp \nu}$. The event selection requires $\left|\vec{p}_{\perp}^{\text {miss }}\right|>25 \mathrm{GeV}$, which effectively removes all DIS NC and photoproduction background. The remaining contamination from cosmic rays and halo muons is eliminated by special filter programs. A total of 14 charged-current events is observed for an integrated luminosity of $\mathcal{L}=348 \pm 17 n b^{-1}$. The efficiencies are essentially determined from the abundant $\mathrm{NC}$ data sample, discarding the scattered electron, and applying the same criteria as for the $\mathrm{CC}$ analysis.

The charged-current cross section for the reaction $e p \rightarrow \nu X$ and neutrino momenta $p_{\perp}>25 \mathrm{GeV}$ is measured as

$$
\sigma^{C C}\left(p_{\perp}>25 \mathrm{GeV}\right)=55 \pm 15 \text { (stat) } \pm 6 \text { (sys) } p b
$$

which can be compared with the expectation of $\sigma_{\text {theor }}^{C C}\left(p_{\perp}>25 \mathrm{GeV}\right)=40.9 \mathrm{pb}$.

The sensitivity of the predicted cross section to a propagator $\left(1+Q^{2} / M_{\text {prop }}^{2}\right)^{-2}$ is shown in Fig. 15. The measurement is consistent with a propagator mass of the 
Figure 15: The charged-current cross section as a function of the propagator mass $M_{\text {prop }}$ (thin line). The dashed line indicates the asymptotic case $M_{\text {prop }}=\infty$. The shaded region represents the measured cross section.

known $W$ resonance, ${ }^{30} M_{W}=80.22 \mathrm{GeV}$, and definitely excludes the asymptotic case $M_{\text {prop }}=\infty$. With the high energy provided by the HERA ep collider, the effect of the $W$ propagator in deep inelastic charged current interactions is visible for the first time.

The measured charged-current cross section has been converted to an equivalent $\nu N$ cross section, shown in Fig. 16. The extrapolation of $p_{\perp}$ to zero and the contribution of the relevant flavors necessarily reduces the sensitivity to the propagator ( $c f$. Fig. 15).

A comparison of the neutral and charged current cross sections with a transverse momentum of the outgoing lepton of $p_{\perp}>25 \mathrm{GeV}$, i.e., $Q^{2}>625 \mathrm{GeV}^{2}$, yields

$$
\frac{\sigma^{N C}\left(p_{\perp}>25 \mathrm{GeV}\right)}{\sigma^{C C}\left(p_{\perp}>25 \mathrm{GeV}\right)}=7.2 \pm 2.1 \text { (stat) } \pm 1.2 \text { (sys) } .
$$

Both cross sections are expected to become of similar magnitude at $Q^{2} \sim M_{W}^{2}$.

\section{Physics with Almost-Real Photons}

Photoproduction is characterized by an electron scattered under a large angle into the backward region of the detector. For tagged electrons, the negative mass 
Figure 16: Energy dependence of the charged-current cross section from low energy $\nu N$ experiments $(+)$. The $\mathrm{H} 1$ measurement $(\bullet)$ has been converted to a $\nu N$ cross section and corresponds to a fixed target energy of $\sim 50 \mathrm{TeV}$. The dashed line indicates a linear extrapolation of the $\nu N$ data; the full line includes the $W$ propagator effect.

squared of the virtual photon, $Q^{2}$, is close to zero and the photon can be considered as almost real. This offers the possibility to study collisions of almost real photons with protons. The total $\gamma p$ cross section has been measured ${ }^{31}$ as

$$
\sigma_{\text {tot }}^{\gamma p}=156 \pm 2 \text { (stat) } \pm 18 \text { (sys) } \mu b
$$

at an average center-of-mass energy of $\left\langle W_{\gamma p}\right\rangle=197 \mathrm{GeV}$.

In most cases, $\gamma p$ scattering proceeds as a soft peripheral collision via vector meson dominance as illustrated in Fig. 10(a), i.e., the photon behaves like a hadron. More insight in the structure of the photon can be obtained by the study of hard $\gamma p$ interactions leading to two or more jets. Generically, one distinguishes between direct processes with $x_{\gamma}=1$, where the photon carries the full fractional momentum $x_{\gamma}$ and interacts pointlike with a parton of the proton [Fig. 17(a)], 
and resolved processes with $x_{\gamma}<1$, where a constituent of the photon interacts with a parton of the proton and an additional photon remnant should be observed [Figs. $17(\mathrm{~b})$ and $17(\mathrm{c})$ ]. Note that $\gamma p$ scattering is more sensitive to the gluon content of the photon, while $\gamma \gamma$ scattering probes the quark content of the photon.

Figure 17: Examples of quasireal photon-proton interactions. (a) Direct photon process $\left(x_{\gamma}=1\right)$; (b) and (c) resolved photon processes $\left(x_{\gamma}<1\right)$.

\subsection{The Structure of the Photon}

In order to study the structure of the photon in hard $\gamma p$ scattering, tagged events with at least two jets of cone radius $R=1$ with a transverse energy of $E_{\perp}^{j e t}>7 \mathrm{GeV}$ within the pseudorapidity range $-0.2<\eta^{j e t}<2.5$ have been selected, which give access to parton momenta $0.03<x_{\gamma}<1$. The momentum fraction $x_{\gamma}$ of the photon can be reconstructed via

$$
x_{\gamma} \simeq \frac{E_{\perp 1} e^{-\eta_{1}}+E_{\perp 2} e^{-\eta_{2}}}{2 E_{\gamma}} .
$$

The $x_{\gamma}$ distribution unfolded for detector effects is presented in Fig. 18(a). The data show a distinct enhancement around $x_{\gamma} \sim 1$, which is an indication of the direct component of $\gamma p$ interactions. The measurements are compared to the PYTHIA ${ }^{3}$ expectations for the direct component and the quark part of the resolved photon contribution using the GRV-LO ${ }^{14}$ structure function. The sum of both components gives a good description of the data for $x_{\gamma}>0.2$. The excess of events at lower $x_{\gamma}$ values can be attributed to the gluon content of the photon. The resulting gluon density in the photon is shown in Fig. 18(b). Despite the still large errors, the data prefer a gluon density rising towards low $x_{\gamma}$ and already discriminate between different parametrizations. ${ }^{14,32}$ 
Figure 18: (a) The $x_{\gamma}$ distribution compared to PYTHIA expectations of the direct component (dashed line) and the quark part of the resolved contribution (full line). (b) The gluon density in the photon compared with several models.

\subsection{Hard Diffractive $\gamma p$ Scattering}

As mentioned above, a large part of the $\gamma p$ cross section is due to diffractive scattering between a vector meson and a Pomeron in the proton. The hard component of diffractive $\gamma p$ scattering can be studied by searching for events with a large rapidity gap between the hadronic final state and the proton remnant, ${ }^{33}$ similar to the DIS analysis in Sec. 4.4.

Figure 19 shows the distribution of events as a function of the maximum pseudorapidity $\eta_{\max }$ at which an energy $E_{\min }>0.4 \mathrm{GeV}$ is deposited in the detector. The majority of events deposit some energy close to the detector acceptance limit around $\eta_{L A r} \sim 3.65$. However, there is a long tail towards small $\eta_{\max }$ values having a large empty phase space between the proton and the hadronic final state. The nondiffractive part of the PYTHIA model badly fails to describe the data. Only the inclusion of photon diffractive dissociation gives a good description of the observed $\eta_{\max }$ spectrum.

In the following, diffractive $\gamma p$ events are defined by requiring a large rapidity gap $\eta_{\max }<1.5$. The remaining nondiffractive contamination is estimated to be negligible.

In Fig. 20, the $p_{\perp}$ spectrum of charged particles in the range $-1.5<\eta<1.5$ is presented. The distribution shows an exponential fall at low transverse momenta 
Figure 19: The $\eta_{\max }$ distribution of photoproduction events compared with the PYTHIA model of nondiffractive (nd) and photon-diffractive dissociation (sd).

with a long tail extending up to $p_{\perp} \sim 5 G e V^{2}$. A similar shape of the $p_{\perp}$ spectrum has been observed in the whole inclusive photoproduction event sample, ${ }^{34}$ where the tail has been interpreted as being due to hard $\gamma p$ scattering. A comparison with models shows that PYTHIA with soft diffraction is able to describe the exponential fall of the bulk of the data at low $p_{\perp}$ but clearly cannot account for the long tail, while the hard diffractive model $\mathrm{POMPYT}^{3}$ reproduces the high $p_{\perp}$ behavior. The sum of both models is in good agreement with the data. The need of a diffractive hard scattering model can be taken as evidence for hard scattering at the parton level in photon diffraction.

Another signature of hard diffractive $\gamma p$ scattering is the observation of jets. A search for jets with a transverse energy of $E_{\perp}^{j e t}>4 \mathrm{GeV}$ within the pseudorapidity range $-1<\eta^{j e t}<1.5$ has been performed. The $\eta_{\max }$ distribution of all $\gamma p$ events containing at least one jet is shown in Fig. 21. The same characteristics as in Fig. 19 are observed: a clear signal of events with $\eta_{\max }<2$, which cannot be explained by the nondiffractive PYTHIA model. Hence, diffractive $\gamma p$ events exhibit jet features similar to the nondiffractive data. The inclusion of the 
Figure 20: Transverse momentum distribution of charged particles in diffractive $\gamma p$ events with a large rapidity gap compared to expectations from a soft diffractive model (PYTHIA, dash-dotted line) and a hard diffractive model (POMPYT, dashed line).

POMPYT model gives a fair description of the data, with a slight preference for a hard gluon distribution in the Pomeron, $x g(x) \sim x(1-x)$, over a soft one, $x g(x) \sim(1-x)^{5}$.

The diffractive data sample with a large rapidity gap $\eta_{\max }<1.5$ and a total transverse energy $E_{T}^{e v t}>5 \mathrm{GeV}$ contains about $7.1 \%$ single-jet events and $1.2 \%$ two-jet events. The jet profiles in the central region are shown in Fig. 22 and are compared to those jet events without a large rapidity gap. The observed profiles are similar except at large $\Delta \eta>1$, corresponding to the region required to be devoid of energy in the rapidity gap selection. The jet profiles as well as the $E_{T}^{j e t}$ and $\eta^{j e t}$ distributions for large rapidity gap diffractive $\gamma p$ events are well-described by the POMPYT model.

In conclusion, both the inclusive $p_{\perp}$ spectrum and the jet analysis show evidence for hard diffractive $\gamma p$ scattering between partons in the photon and partons in the Pomeron. 
Figure 21: The $\eta_{\max }$ distribution of $\gamma p$ events containing at least one jet compared with the nondiffractive (nd) PYTHIA model and POMPYT with a hard- and a soft-gluon structure function.

Figure 22: Jet profiles of diffractive events with and without large rapidity gaps for $4 \mathrm{GeV}<E_{T}^{j e t}<6 \mathrm{GeV}$ and $\left|\eta^{j e t}\right|<0.5$. The histogram represents the POMPYT model. 
Figure 23: (a) Mass spectrum $M_{\ell^{+} \ell^{-}}$for $e p \rightarrow \ell^{+} \ell^{-} X$ compared to QED expectation (shaded area). The curve is a fit to the $J / \psi$ signal. (b) $d \sigma / d p_{T}^{2}$ for $e p \rightarrow J / \psi X$ compared with model predictions. The thick line is a fit to the data.

\subsection{Photoproduction of $J / \psi$ Mesons}

Heavy quark production by virtual or real photons is a sensitive tool to study strong interaction physics in a transition region between perturbative and nonperturbative QCD. Since the energy scale, set by the heavy quark mass, is high enough to provide a sufficiently low strong coupling $\alpha_{s}$, a perturbative approach is justified. On the other hand, perturbation theory breaks down if long range "soft" interactions between the heavy quarks and the proton dominate. The photoproduction of $J / \psi$ vector mesons is ideal to study this transition regime in the charm sector.

In the analysis ${ }^{35}$ of the reaction $e p \rightarrow J / \psi X$, the scattered electron remains undetected, limiting the virtuality of the photon to $Q^{2} \leq 4 \mathrm{GeV}^{2}$ and the $\gamma p$ cms energy to $30 \mathrm{GeV}<W_{\gamma p}<180 \mathrm{GeV}$. J/ $\psi$ candidates are identified by their leptonic decay modes, requiring two oppositely charged electrons or muons and nothing else in the detector. These criteria select true elastic $J / \psi$ production contaminated by an unknown admixture of proton dissociation with unobserved fragments remaining in the beam pipe.

The mass spectrum of $e^{+} e^{-}$and $\mu^{+} \mu^{-}$pairs is shown in Fig. 23(a). A clear $J / \psi$ signal at $M_{J / \psi}=3.10 \mathrm{GeV}$ is observed over a smooth background from QED lepton pair production. A fit to the data around $\pm 0.225 \mathrm{GeV}$ of the nominal 
$J / \psi$ mass yields $19 \pm 5$ muon pairs $\left(\mathcal{L}=259 n b^{-1}\right)$ and $7 \pm 3$ electron pairs $\left(\mathcal{L}=284 n b^{-1}\right)$. The result for the cross section is

$$
\sigma(e p \rightarrow J / \psi X)=11.9 \pm 2.5(\text { stat }) \pm 3.0(\text { sys }) n b
$$

The e $p$ cross section can be converted into a $\gamma p$ cross section after unfolding the photon flux. The total photoproduction cross section for $J / \psi$ mesons is

$$
\sigma(\gamma p \rightarrow J / \psi X)=57 \pm 12(\text { stat }) \pm 14(\text { sys }) n b
$$

at an average center-of-mass energy of $\left\langle W_{\gamma p}\right\rangle=90 \mathrm{GeV}$.

Figure 24: Total cross section $(\bullet)$ for $\gamma p \rightarrow J / \psi X$ compared with other experiments and various model predictions.

A comparison with earlier low energy experiments is presented in Fig. 24. The $J / \psi$ photoproduction cross section still rises at HERA energies, but its interpretation is not obvious due to the unknown inelastic contribution, present to some extent in most experiments. The $\mathrm{H} 1$ selection accepts events with an elasticity parameter $z=E_{J / \psi} / E_{\gamma} \geq 0.95$, where the energies are measured in the proton rest system. The VMD model of PYTHIA predicts an elastic contribution of $\sim 50 \%$ at HERA energies and falls below the measurement. The same is true everywhere 
for the elastic part of the QCD-inspired model of Jung et al. ${ }^{3}$ If inelastic contributions with $z>0.95$ are included, the $\mathrm{H} 1$ cross section can be reproduced using the MRS $\mathrm{D}^{-}$structure function. A flat gluon distribution as in MRS $\mathrm{D}^{0}$ lowers the prediction by a factor of $\sim 1.6$, which emphasizes the sensitivity of charm production to the gluon content in the proton.

More information on the characteristics of $J / \psi$ production can be obtained from the dependence on the momentum transfer $t$ from the proton to the $J / \psi$, which can be approximated by $t=\left(p_{\gamma}-p_{J / \psi}\right)^{2} \simeq-p_{T}^{2}$. The differential cross section $d \sigma / d p_{T}^{2}$ is displayed in Fig. 23(b). The tail towards larger $p_{T}^{2}$ is due to events with finite values of $Q^{2}$. An exponential fit to the data below $p_{T}^{2}<$ $0.75 \mathrm{GeV}^{2}$ yields a slope of $b=-(4.7 \pm 1.9) \mathrm{GeV}^{-2}$. In models with pure elastic diffraction, a steeper $p_{T}^{2}$ dependence of $b \simeq-8 \mathrm{GeV}^{-2}$ is expected, while the inclusion of inelastic contributions reproduces the data fairly well.

Future investigations with high statistics will concentrate on determining the inelastic contribution to $J / \psi$ production. Furthermore, the detection of heavy flavors through their semileptonic decays or $D^{*}$ production will allow another measurement of the gluon density in the proton.

\section{Beyond the Standard Model}

The large, available, center-of-mass energy at HERA has been explored to search for physics beyond the Standard Model. Signals for new phenomena can be discovered either directly or indirectly.

Common to all direct searches is the s channel formation of new heavy resonances, such as heavy leptons, leptoquarks, leptogluons, and squarks, showing up as a peak in the mass distribution at $M=\sqrt{x s}$. The production cross section for a heavy particle $H$ is given by

$$
\sigma(e p \rightarrow H X)=\frac{4 \pi^{2}}{s}(2 J+1) \frac{\Gamma}{M} \mathcal{B} f_{i / p}\left(M^{2} / s\right)
$$

where $J$ is the spin of the particle $H$ and $f_{i / p}(x)$ is the density of partons $i$ in the proton with momentum fraction $x=M^{2} / s$. The width $\Gamma$ and the decay branching ratio $\mathcal{B}$ depend on specific models for the coupling to the new particle.

The search for new bosons or eq compositeness can be considerably extended beyond the kinematic production limit of HERA through the study of indirect effects from a virtual particle exchange. Such virtual effects are conveniently 
described by contact interactions ${ }^{36}$ and show up as deviations from the Standard Model at high $Q^{2}$ values.

The various analyses are based on an integrated luminosity of $\mathcal{L}=320 \div$ $528 n b^{-1}$. All model-independent limits on masses and couplings are quoted at a 95\% confidence level.

Heavy leptons are searched for in the channels ${ }^{37}$

$$
\begin{gathered}
e^{*} \rightarrow e \gamma, \quad e Z, \quad \nu W \\
\nu^{*} \rightarrow \nu \gamma, \quad \nu Z, \quad e W
\end{gathered}
$$

with the subsequent decays of the gauge bosons $Z$ and $W$ into lepton or quark pairs. No deviations from QED Compton scattering and standard DIS are observed for masses between $10 \mathrm{GeV}$ and $225 \mathrm{GeV}$. Therefore, cross sections for new heavy lepton production of

$$
\begin{aligned}
\sigma\left(e p \rightarrow e^{*}\left(\nu^{*}\right) X\right) & >\mathcal{O}(10 p b) \\
\text { for masses } M_{e^{*}\left(\nu^{*}\right)} & <225 \mathrm{GeV}
\end{aligned}
$$

can be ruled out.

Leptoquarks are color-triplet bosons of spin 0 or 1 carrying lepton $(L)$ and baryon $(B)$ numbers. The new quantum number $F=L+3 B$ can take values of $F=2$ for $\left(e^{-} q\right)$ leptoquarks and $F=0$ for $\left(e^{-} \bar{q}\right)$ leptoquarks. They are classified $^{38}$ according to their spin and isospin quantum numbers. The partial decay widths $\Gamma$ are related to the coupling constants $\lambda$ by $\Gamma_{S}=\left(\lambda^{2} / 16 \pi\right) M$ for scalar and $\Gamma_{V}=\left(\lambda^{2} / 24 \pi\right) M$ for vector leptoquarks. The decay topologies into a lepton + jet are indistinguishable from neutral and charged DIS events, but the angular decay distributions differ due to the spin of the leptoquarks.

The observed mass spectra ${ }^{39}$ are fully compatible with the expectations from Standard Model DIS events. The derived model independent limits on the couplings $\lambda$ as a function of the scalar and vector leptoquark masses are presented in Fig. 25. There is a considerable improvement over previous measurements. ${ }^{40,41}$ For low masses, where the quark densities are high, very small couplings are accessible, while for masses close to the kinematic limit, the quark densities become so low that couplings of $\lambda \sim 1$ are necessary to get observable cross sections. As expected, better limits are obtained for leptoquarks coupling to quarks than for leptoquarks coupling to antiquarks. 
Figure 25: Upper limits (95\% confidence level) on couplings $\lambda$ as a function of mass for scalar and vector leptoquarks; (a) and (b) show $\left(e^{-} q\right)$ leptoquarks with $F=2$, while (c) and (d) show $\left(e^{-} \bar{q}\right)$ leptoquarks with $F=0$. The additional lines in (b) and (d) represent the results of indirect searches from a contact-term analysis. 
Indirect searches for certain vector leptoquarks with enhanced couplings to $u$ quarks from a contact-term analysis complement the direct searches. They provide more stringent bounds at masses approaching or exceeding the HERA center-of-mass energy and scale as $M_{L Q} / \lambda$.

Assuming a coupling of electromagnetic strength $(\lambda=\sqrt{4 \pi \alpha}=0.3)$ for the direct searches, the following mass limits for leptoquarks can be set

$$
\begin{array}{llll}
\text { direct searches } & \text { scalars } & M_{S}>135 \div 235 \mathrm{GeV} & \lambda=0.3 \\
& \text { vectors } & M_{V}>145 \div 230 \mathrm{GeV} & \lambda=0.3 \\
\text { indirect searches } & \text { vectors } & M_{V}>300 \div 530 \mathrm{GeV} & \lambda=1 .
\end{array}
$$

For comparison, the $\mathrm{D} \emptyset$ experiment ${ }^{42}$ gives lower mass limits of $116(130) \mathrm{GeV}$ for scalar leptoquarks and 189 (195) $\mathrm{GeV}$ for vector leptoquarks assuming a branching ratio $\mathcal{B}=0.5(1)$ into electron and quark. The mass limits from hadron colliders are independent of the coupling $\lambda$, because leptoquarks are dominantly produced via a virtual gluon.

Leptogluons appear in composite models as color octet partners of the color singlet leptons. The signature of an electron + jet final state is similar to leptoquarks and neutral current DIS events, but the angular decay distribution is characteristic for a spin $1 / 2$ particle. The production cross section depends on a scale parameter $\Lambda$, which is related to the decay width by $\Gamma=\alpha_{s} M^{3} /\left(4 \Lambda^{2}\right)$. Again, no deviation of the measured mass spectra from the Standard Model expectation is observed. This can be converted into an exclusion limit. Leptogluons of masses $M_{L G}<169 \mathrm{GeV}$ for compositeness scales $\Lambda>1 \mathrm{TeV}$ are ruled out.

Squarks $(\tilde{q})$ are predicted in supersymmetric extensions of the Standard Model as scalar partners of the known quarks. In some supersymmetric models, the $R_{p}$ parity, defined as $R_{p}=(-1)^{3 B+L+2 S}$ with $S$ being the spin, can be violated. $R_{p}$ squark decays are searched for in the decay sequence

$$
\begin{aligned}
\tilde{q} \rightarrow & \tilde{\gamma} q \\
& \hookrightarrow q q^{\prime} e^{ \pm}(\nu) .
\end{aligned}
$$

The striking signature is an apparent flavor-changing neutral current with a positron in the final state in half of the photino decays. The $R_{p}$ coupling between the first generation fermions is denoted as $\lambda_{111}^{\prime}$ and depends on the assumed photino and squark masses.

The absence of a signal can be converted into rejection limits of $\not h_{p}$ squarks for different photino masses, presented in Fig. 26. The limits for $\lambda_{111}^{\prime}$ are similar to 
those for the $S_{0}^{L}$ leptoquark, but here, the gauge decay contributes dominantly at low coupling (i.e., $M_{\tilde{q}} \leq 100 \mathrm{GeV}$ ) and the $\not R_{p}$ decay at masses above $175 \mathrm{GeV}$. Limits for other $\tilde{q}$ decay modes can be derived by multiplying $\lambda_{111}^{\prime}$ with the square root of the appropriate branching ratios.

Figure 26: Upper limits (95\% confidence level) on the coupling $\lambda_{111}^{\prime}$ as a function of squark mass for various photino masses.

Assuming a coupling of electromagnetic strength $\left(\lambda_{111}^{\prime}=0.3\right)$ squarks with masses of $M_{\tilde{q}}>239 \mathrm{GeV}$ in $\not R_{p}$ supersymmetric models can be excluded, with a weak dependence on the assumed photino mass.

For comparison, the dilepton data of the Tevatron experiments can be used to estimate $^{43}$ a mass limit for $\not R_{p}$ squarks of $M_{\tilde{q}}>100 \mathrm{GeV}$.

$e q$ compositeness occurs in models where the fermions are supposed to have a substructure. If they have common constituents, an eq compositeness scale parameter $\Lambda$ can be defined for various chiral couplings (left- and/or right-handed helicities of electrons and/or quarks).

A possible $e q$ compositeness is searched for in a contact-term analysis. Assuming a strong coupling $g^{2} / 4 \pi=1$, lower limits on the compositeness scale for positive and negative interference with the $\gamma$ and $Z$ fields of the Standard Model can be set 


$$
\Lambda_{+}>1.15 \mathrm{TeV} \text { and } \Lambda_{-}>0.75 \mathrm{TeV}
$$

These limits are almost independent of the chiral structure.

Similar studies at $e^{+} e^{-}$and $p \bar{p}$ colliders ${ }^{30}$ yield values roughly a factor of two to three higher.

Although so far no evidence for new phenomena beyond the Standard Model is found, substantially improved limits on couplings and masses for the production of new particles can be given. The direct searches cover almost the entire accessible mass region of HERA. Very promising for future investigations are indirect signatures by virtual boson exchange. The sensitivity to contact-term interactions roughly scales as $(\mathcal{L} s)^{\frac{1}{4}}$ with integrated luminosity $(\mathcal{L})$ and center-of-mass energy $(s)$.

\section{Conclusion}

The first two years of HERA have already provided a wealth of new, interesting physics results concerning many different aspects of lepton nucleon scattering, such as neutral and charged current deep inelastic scattering, photoproduction at high energies, and searches for phenomena beyond the Standard Model. Only a selected part of the analyses carried out at the $\mathrm{H} 1$ experiment could be presented in this paper.

With the anticipated improved performance of the HERA collider, many more data will be collected in the future, allowing us to study in more detail the addressed physics issues of $e p$ scattering.

\section{Acknowledgments}

I am grateful to my colleagues from the H1 Collaboration for the many valuable discussions. I would like to thank A. DeRoeck for a careful reading of the manuscript. Finally, my thanks go to the organizers of the 1994 SLAC Summer Institute for providing such a stimulating and most pleasant atmosphere. 


\section{References}

[1] H1 Collaboration, I. Abt et al., "The H1 detector at HERA," DESY 93-103 (1993), submitted to Nucl. Instrum. and Methods.

[2] Proceedings of the "Physics at HERA" Workshop, Vol. 1, DESY, Hamburg (1991), edited by W. Buchmüller and G. Ingelman.

[3] Monte Carlo generators, "Physics at HERA," (see Ref. 2) Vol. 3 (1991).

[4] A. D. Martin, W. J. Stirling, and R. G. Roberts, Phys. Lett. B 306, 145 (1993); ibid. B 309, 492 (1993).

[5] B. Löhr, "Recent results from the ZEUS experiment at HERA," these proceedings.

[6] NMC Collaboration, P. Amaudruz et al., Phys. Lett. B 295, 159 (1992).

[7] BCDMS Collaboration, A. C. Benvenuti et al., Phys. Lett. B 223, 485 (1989).

[8] H1 Collaboration, I. Abt et al., Nucl. Phys. B 407, 515 (1993).

[9] ZEUS Collaboration, M. Derrick et al., Phys. Lett. B 316, 412 (1993).

[10] J. Botts et al., Phys. Lett. B 304, 159 (1993).

[11] A. Donnachie and P. Landshoff, M/C-th 93/11, DAMTP 92-23.

[12] V. N. Gribov and L. N. Lipatov, Sov. J. Nucl. Phys. 15, 438 (1972);

G. Altarelli and G. Parisi, Nucl. Phys. B 126, 298 (1077);

Y. L. Dokshitzer, Sov. Phys. JETP 46, 641 (1977).

[13] E. A. Kuraev, L. N. Lipatov, and V. S. Fadin, Sov. Phys. JETP 45, 199 (1977);

Y. Y. Balitsky and L. N. Lipatov, Sov. J. Nucl. Phys. 28, 822 (1978).

[14] M. Glück, E. Reya, and A. Vogt, Z. Phys. C 53, 127 (1992); Phys. Lett. B 306, 391 (1993).

[15] L. F. Abbott, W. B. Atwood, and R. M. Barnett, Phys. Rev. D 22, 582 (1980).

[16] K. Prytz, Phys. Lett. B 311, 286 (1993).

[17] H1 Collaboration, I. Abt et al., Z. Phys. C 94, 377 (1994).

[18] K. Golec-Biernat, J. Kwieciński, A. D. Martin, and P. J. Sutton, Phys. Lett. B 335, 220 (1994). 
[19] A. H. Mueller, J. Phys. G 19, 1463 (1993).

[20] A. D. Martin, J. Kwieciński, and P. J. Sutton, Nucl. Phys. B (Proc. Suppl.) 298, 67 (1992).

[21] H1 Collaboration, T. Ahmed et al., preprint DESY 94-133 (1994), submitted to Nucl. Phys. B.

[22] ZEUS Collaboration, M. Derrick et al., Phys. Lett. B 315, 481 (1993).

[23] W. Bartel, A. DeRoeck, in Proceedings of the Europhysics Conf. on HEP, Marseille, France (1993); J. Dainton, in Proceedings of the XVI International Symposium on Lepton Photon Interactions, Cornell, Ithaca, USA (1993), edited by P. Drell and D. Rubin.

[24] H. Jung, "The Monte Carlo generator RAPGAP," preprint DESY 93-182 (1993), submitted to Comput. Phys. Commun.

[25] R. Nisius, Ph.D. thesis, RWTH Aachen (1994); H1 Collaboration, T. Ahmed et al., to be published.

[26] D. Graudenz, "PROJET program manual," unpublished.

[27] S. Bethke and J. E. Pilcher, Ann. Rev. Nucl. Sci. 42, 251 (1992).

[28] D. Haidt and H. Pietschmann, Landoldt-Börnstein New Series I/10, Springer (1988).

[29] H1 Collaboration, T. Ahmed et al., Phys. Lett. B 323, 241 (1994).

[30] Particle Data Group, Phys. Rev. D 50, 1173 (1994).

[31] H1 Collaboration, T. Ahmed et al., Phys. Lett. B 299, 374 (1993).

[32] H. Abramowicz, K. Charchula, and A. Levy, Phys. Lett. B 269, 458 (1991).

[33] H1 Collaboration, T. Ahmed et al., to be submitted to Phys. Lett. B.

[34] H1 Collaboration, I. Abt et al., Phys. Lett. B 328, 176 (1994).

[35] H1 Collaboration, T. Ahmed et al., Phys. Lett. B 338, 507 (1994).

[36] P. Haberl, F. Schrempp, and H.-U. Martyn, "Physics at HERA," (see Ref. 2) Vol. 2, 1133 (1991).

[37] H1 Collaboration, T. Ahmed et al., preprint DESY 94-138 (1994), submitted to Phys. Lett. B.

[38] B. Schrempp, "Physics at HERA," (see Ref. 2) Vol. 2, 1034 (1991). 
[39] H1 Collaboration, T. Ahmed et al., preprint DESY 94-154 (1994), submitted to Phys. Lett. B.

[40] H1 Collaboration, I. Abt et al., Nucl. Phys. B 396, 3 (1993).

[41] ZEUS Collaboration, M. Derrick et al., Phys. Lett. B 306, 173 (1993).

[42] M. Abolins, "D $\emptyset$ top results, QCD, electroweak and new phenomena physics at Fermilab," these proceedings; D $\emptyset$ Collaboration, S. Abachi et al., Phys. Rev. Lett. 72, 965 (1994); CDF Collaboration, F. Abe et al., Phys. Rev. D 48, 3939 (1993).

[43] D. P. Roy, Phys. Lett. B 283, 270 (1992). 